\title{
Continuous Solutions of Conditional Composite Type Functional Equations
}

\author{
Jacek Chudziak and Zdeněk Kočan
}

\begin{abstract}
It is known that some problems in meteorology and fluid dynamics lead to Gołąb-Schinzel type equations on a restricted domain. Inspired by a question of Professor L. Reich we determine the solutions of conditional composite type functional equations related to the Gołąb-Schinzel equation.
\end{abstract}

Mathematics Subject Classification (2010). 39B12, 39B22.

Keywords. Composite functional equation, Gołąb-Schinzel equation, continuous solutions, restricted domain.

\section{Introduction}

The Gołąb-Schinzel equation

$$
F(x+F(x) y)=F(x) F(y)
$$

is one of the most important composite type functional equations. It has been considered for the first time by Gołąb and Schinzel [13]. Some applications of (1) and its further generalizations can be found e.g. in $[2,4,7,14,20]$ and [23]. For more details concerning Eq. (1) we refer to [1] and to a survey paper [9]. In [18] it has been shown that some problems in meteorology and fluid mechanics lead to Eq. (1) on a restricted domain. Inspired by this fact several authors have considered conditional versions of (1). Continuous solutions $f: \mathbb{R} \rightarrow \mathbb{R}$ of the equation

$$
f(x+f(x) y)=f(x) f(y) \text { for } x, y \in[0, \infty)
$$

have been determined in [3]. Another conditional generalization of (1), namely the equation

$$
f(x+f(x) y)=f(x) f(y) \quad \text { whenever } \quad x, y, x+f(x) y \geq 0
$$


has been considered among others in [10,21,22] and [24]. Some further results concerning conditional Gołąb-Schinzel type functional equations can be found in $[5,6,8,12,25]$ and [26].

During the 14th ICFEI meeting (Bȩdlewo, Poland, 2011) Professor Ludwig Reich posed the question concerning solutions of a conditional version of the following equation

$$
f(x+g(x) y)=f(x) f(y) .
$$

Some aspects of this equation have been studied in [11] and [15-17]. Motivated by the question of Professor L. Reich we determine the solutions of the functional equation

$$
f(x+g(x) y)=f(x) f(y) \quad \text { whenever } \quad x, y, x+g(x) y \geq 0
$$

in the class of pairs $(f, g)$ of continuous functions mapping the interval $[0, \infty)$ into $\mathbb{R}$. As a consequence of our main result we obtain the solutions of the equation

$$
F(x+G(x) y)=F(x) F(y) \text { for } x, y \in[0, \infty)
$$

in the class of pairs $(F, G)$ of continuous functions mapping $\mathbb{R}$ into $\mathbb{R}$. In particular we generalize to some extent the results in [3] and [24].

\section{Auxiliary Results}

We begin with a result which can be easily deduced from [15, Theorem 1].

Proposition 2.1. Assume that $F, G: \mathbb{R} \rightarrow \mathbb{R}$ are continuous functions. Then the pair $(F, G)$ satisfies equation

$$
F(x+G(x) y)=F(x) F(y) \text { for } x, y \in \mathbb{R}
$$

if and only if one of the following possibilities holds:

(i) $F(x)=0$ for $x \in \mathbb{R}$;

(ii) $F(x)=1$ for $x \in \mathbb{R}$;

(iii) $G(x)=1$ for $x \in \mathbb{R}$ and there exists a $c \in \mathbb{R} \backslash\{0\}$ such that $F(x)=$ $e^{c x}$ for $x \in \mathbb{R}$;

(iv) there exist a $c \in \mathbb{R} \backslash\{0\}$ and an $r \in(0, \infty)$ such that

$$
G(x)=\max \{1+c x, 0\} \text { for } x \in \mathbb{R}
$$

and

$$
F(x)=(\max \{1+c x, 0\})^{r} \text { for } x \in \mathbb{R} ;
$$

(v) there exist a $c \in \mathbb{R} \backslash\{0\}$ and an $r \in(0, \infty)$ such that

$$
G(x)=1+c x \quad \text { for } \quad x \in \mathbb{R}
$$

and either

$$
F(x)=|1+c x|^{r} \quad \text { for } \quad x \in \mathbb{R}
$$


or

$$
F(x)=|1+c x|^{r} \cdot \operatorname{sgn}(1+c x) \quad \text { for } \quad x \in \mathbb{R} .
$$

In the sequel we will use the following notation. Given a function $f$ : $[0, \infty) \rightarrow \mathbb{R}$, we set $Z_{f}=\{x \in[0, \infty) \mid f(x)=0\}$. Moreover, if $g:[0, \infty) \rightarrow \mathbb{R}$ and $x \in[0, \infty)$ then by $g^{(x)}$ we denote a function $g^{(x)}:[0, \infty) \rightarrow \mathbb{R}$ given by

$$
g^{(x)}(y)=x+g(x) y \quad \text { for } y \in[0, \infty) .
$$

Remark 2.2. Note that if a pair of functions $(f, g)$, where $f, g:[0, \infty) \rightarrow \mathbb{R}$, satisfies Eq. (4) then

$$
f\left(g^{(x)}(y)\right)=f(x) f(y) \quad \text { whenever } \quad x, y, g^{(x)}(y) \geq 0 .
$$

In particular, for every $x, y \in[0, \infty)$ such that $g^{(x)}(y) \geq 0$ there holds

$$
g^{(x)}(y) \in Z_{f} \quad \text { if and only if } \quad\left(x \in Z_{f} \text { or } y \in Z_{f}\right) .
$$

Moreover $g^{(x)}(0)=x$ for every $x \in[0, \infty)$. Eq. (4).

Now we prove some preliminary results concerning the solutions of

Lemma 2.3. Assume that a pair of functions $(f, g)$, where $f, g:[0, \infty) \rightarrow \mathbb{R}$, satisfies Eq. (4). Then:

(a) $f(0) \in\{0,1\}$,

(b) if $f(0)=0$ then $f(x)=0$ for $x \in[0, \infty)$,

(c) either $f(x)=1$ for $x \in[0, \infty)$, or $Z_{g} \subset Z_{f}$.

Proof. (a) It is enough to apply (4) with $x=y=0$.

(b) Assume that $f(0)=0$. Then, putting in (4) $y=0$, we get the assertion.

(c) Assume that $f\left(x_{1}\right) \neq 1$ for some $x_{1} \in[0, \infty)$. Since the case where $Z_{g}=\emptyset$ is trivial, assume that $Z_{g} \neq \emptyset$ and fix an $x_{0} \in Z_{g}$. Then, applying (4) with $x=x_{0}$ and $y=x_{1}$, we get $f\left(x_{0}\right)=f\left(x_{0}\right) f\left(x_{1}\right)$. As $f\left(x_{1}\right) \neq 1$, this means that $x_{0} \in Z_{f}$.

Lemma 2.4. Assume that $f, g:[0, \infty) \rightarrow \mathbb{R}$ are continuous functions and $f(0)=1$. If the pair $(f, g)$ satisfies $E q$. (4) and $Z_{f}$ contains at least two elements then $Z_{f}=[z, \infty)$ with some $z \in(0, \infty)$.

Proof. Assume that $(f, g)$ satisfies $(4)$ and $Z_{f}$ contains at least two elements. First we show that $Z_{f}$ is an interval. For the proof by contradiction, suppose that it is not true. Note that as $f$ is continuous, $Z_{f}$ is closed. Thus, there exist $z_{1}, z_{2} \in Z_{f}$ such that $z_{1}<z_{2}$ and

$$
\left(z_{1}, z_{2}\right) \cap Z_{f}=\emptyset \text {. }
$$

In view of Lemma $2.3(c)$, from (11) follows that $\left(z_{1}, z_{2}\right) \cap Z_{g}=\emptyset$. Since $g$ is continuous, this means that either $g(x)<0$ for $x \in\left(z_{1}, z_{2}\right)$, or $g(x)>0$ for $x \in\left(z_{1}, z_{2}\right)$. In the first case, for every $x \in\left(z_{1}, z_{2}\right), g^{(x)}$ is strictly decreasing, whence $g^{(x)}\left(z_{2}\right)<g^{(x)}(0)=x<z_{2}$. Furthermore, as $z_{2} \in Z_{f}$, in view of 
Remark 2.2, for every $x \in\left(z_{1}, z_{2}\right)$, either $g^{(x)}\left(z_{2}\right)<0$ or $g^{(x)}\left(z_{2}\right) \in Z_{f}$. Thus, for every $x \in\left(z_{1}, z_{2}\right), g^{(x)}\left(z_{2}\right) \leq z_{1}$ and so $g(x) \leq \frac{z_{1}-x}{z_{2}}$. Since $g$ is continuous, this implies that $g\left(z_{2}\right)=\frac{z_{1}-z_{2}}{z_{2}}<0$. Thus $\frac{z_{1}-z_{2}}{2 g\left(z_{2}\right)}>0$ and $z_{2}+g\left(z_{2}\right) \frac{z_{1}-z_{2}}{2 g\left(z_{2}\right)}=$ $\frac{z_{1}+z_{2}}{2}>0$, so taking in (4) $x=z_{2}$ and $y=\frac{z_{1}-z_{2}}{2 g\left(z_{2}\right)}$, we obtain that $f\left(\frac{z_{1}+z_{2}}{2}\right)=0$. Hence $\frac{z_{1}+z_{2}}{2} \in Z_{f}$, which contradicts (11).

If $g(x)>0$ for $x \in\left(z_{1}, z_{2}\right)$ then arguing as previously we get $g(x) \geq \frac{z_{2}-x}{z_{1}}$ for $x \in\left(z_{1}, z_{2}\right)$. Therefore $g\left(z_{1}\right)>0$ and so, as $\frac{z_{2}-z_{1}}{2 g\left(z_{1}\right)}>0$ and $z_{1}+g\left(z_{1}\right) \frac{z_{2}-z_{1}}{2 g\left(z_{1}\right)}=$ $\frac{z_{1}+z_{2}}{2}>0$, putting in (4) $x=z_{1}$ and $y=\frac{z_{2}-z_{1}}{2 g\left(z_{1}\right)}$, we obtain $\frac{z_{1}+z_{2}}{2} \in Z_{f}$ which again contradicts (11).

In this way we have proved that $Z_{f}$ is an interval. Let $z:=\min Z_{f}$ and $z_{\max }:=\sup Z_{f}$. Suppose that $z_{\max }<\infty$. Then $Z_{f}=\left[z, z_{\max }\right]$. Moreover, according to Remark $2.2, g^{(x)}\left(z_{\max }\right) \leq z_{\max }$ for $x \in\left(z_{\max }, \infty\right)$. Hence $g(x) \leq$ $1-\frac{x}{z_{\max }}<0$ for $x \in\left(z_{\max }, \infty\right)$. This means that, for every $x \in\left(z_{\max }, \infty\right)$, $g^{(x)}$ is strictly decreasing. Thus

$$
g^{(x)}(y)<g^{(x)}(0)=x \quad \text { for } x \in\left(z_{\max }, \infty\right), y \in(0, \infty) .
$$

Note also that

$$
z_{\max }<g^{(x)}(y) \text { for } x \in\left(z_{\max }, \infty\right), y \in(0, z) .
$$

Otherwise, as $g^{(x)}$ is continuous for $x \in[0, \infty)$ and $z_{\max }<x=g^{(x)}(0)$ for $x \in\left(z_{\max }, \infty\right)$, we would have $g^{(x)}(y)=z_{\max }$ for some $x \in\left(z_{\max }, \infty\right)$ and $y \in(0, z)$, which contradicts (10). Now, from (12) and (13) we derive that $g^{(x)}(y) \in\left(z_{\max }, x\right)$ for $x \in\left(z_{\max }, \infty\right), y \in(0, z)$. Since $g^{(x)}$ is continuous for $x \in[0, \infty)$ this means that $g^{(x)}(z) \in\left[z_{\max }, x\right] \subset[0, \infty)$ for $x \in\left(z_{\max }, \infty\right)$. Furthermore, making use of $(10)$, we get $g^{(x)}(z) \in Z_{f}=\left[z, z_{\max }\right]$ for $x \in$ $\left(z_{\max }, \infty\right)$. Thus $g^{(x)}(z)=z_{\max }$ for $x \in\left(z_{\max }, \infty\right)$, whence $g(x)=\frac{z_{\max }-x}{z}$ for $x \in\left(z_{\max }, \infty\right)$. Therefore, taking $x_{0}:=z_{\max }+\frac{1}{2} z$ and $y_{0}:=2 z_{\max }-z$, we have $x_{0}, y_{0}>z_{\max }>0$ and $g^{\left(x_{0}\right)}\left(y_{0}\right)=x_{0}+g\left(x_{0}\right) y_{0}=z_{\max }+\frac{1}{2} z-\frac{1}{2}\left(2 z_{\max }-z\right)=z$. Since $Z_{f}=\left[z, z_{\max }\right]$, this contradicts (10). Consequently $z_{\max }=\infty$ and so $Z_{f}=[z, \infty)$.

\section{Main Results}

The next theorem is the main result of the paper.

Theorem 3.1. Assume that $f, g:[0, \infty) \rightarrow \mathbb{R}$ are continuous functions. Then the pair $(f, g)$ satisfies Eq. (4) if and only if one of the subsequent possibilities holds:

(i) $f(x)=0$ for $x \in[0, \infty)$;

(ii) $f(x)=1$ for $x \in[0, \infty)$;

(iii) $g(x)=1$ for $x \in[0, \infty)$ and there exists a $c \in \mathbb{R} \backslash\{0\}$ such that $f(x)=$ $e^{c x}$ for $x \in[0, \infty)$; 
(iv) there exist a $c \in(0, \infty)$ and an $r \in \mathbb{R} \backslash\{0\}$ such that

$$
g(x)=1+c x \quad \text { for } \quad x \in[0, \infty)
$$

and

$$
f(x)=(1+c x)^{r} \quad \text { for } \quad x \in[0, \infty) \text {; }
$$

(v) there exist a $c \in(-\infty, 0)$ and an $r \in(0, \infty)$ such that $g$ is of the form (14) and either

$$
f(x)=|1+c x|^{r} \quad \text { for } \quad x \in[0, \infty)
$$

or

$$
f(x)=|1+c x|^{r} \cdot \operatorname{sgn}(1+c x) \quad \text { for } \quad x \in[0, \infty)
$$

(vi) there exist a $c \in(-\infty, 0)$, an $r \in(0, \infty)$ and a continuous function $\phi:\left[-\frac{1}{c}, \infty\right) \rightarrow[0, \infty)$ with $\phi\left(-\frac{1}{c}\right)=0$ such that

$$
g(x)= \begin{cases}1+c x & \text { for } x \in\left[0,-\frac{1}{c}\right) \\ \phi(x) & \text { for } x \in\left[-\frac{1}{c}, \infty\right)\end{cases}
$$

and

$$
f(x)=(\max \{1+c x, 0\})^{r} \quad \text { for } \quad x \in[0, \infty) .
$$

Proof. Assume that the pair $(f, g)$ satisfies (4). Then, in view of Lemma 2.3( $a)$, $f(0) \in\{0,1\}$. Moreover, if $f(0)=0$ then applying Lemma 2.3(b), we get (i). So, assume that $f(0)=1$. Then, according to Lemma 2.4, one of the following three cases holds:

(I) $Z_{f}=\emptyset$;

(II) $Z_{f}=\{z\}$ with some $z \in(0, \infty)$;

(III) $Z_{f}=[z, \infty)$ with some $z \in(0, \infty)$.

Case (I) If $f(x)=1$ for $x \in[0, \infty)$ then (ii) is valid. Suppose that $f\left(x_{1}\right) \neq 1$ for some $x_{1} \in(0, \infty)$. Then, in view of Lemma $2.3(c), Z_{g}=\emptyset$. Since $g$ is continuous this implies that either $g(x)<0$ for $x \in[0, \infty)$, or $g(x)>0$ for $x \in[0, \infty)$. In the first case, taking $y=\frac{x_{1}}{1-g\left(x_{1}\right)}$, we have $y>0$ and $x_{1}+g\left(x_{1}\right) y=y>0$. Hence, making use of (4), we get $f(y)=f\left(x_{1}+g\left(x_{1}\right) y\right)=$ $f\left(x_{1}\right) f(y)$. As $Z_{f}=\emptyset$ and $f\left(x_{1}\right) \neq 1$, this yields a contradiction. Thus $g(x)>0$ for $x \in[0, \infty)$, so in view of $(4)$, we get

$$
f(x+g(x) y)=f(x) f(y) \text { for } x, y \in[0, \infty) .
$$

If $g(x)=1$ for $x \in[0, \infty)$ then we have $f(x+y)=f(x) f(y)$ for $x, y \in[0, \infty)$. Hence, as $f$ is continuous, not identically equal to 1 and $Z_{f}=\emptyset$, applying [19, p. 311, Theorem 4] and [27, Corollary 2], we obtain (iii).

Assume that $g\left(x_{0}\right) \neq 1$ for some $x_{0} \in[0, \infty)$ and put $k:=g\left(x_{0}\right)$ and $a:=f\left(x_{0}\right)$. Suppose that $k \in(0,1)$. Then $\frac{x_{0}}{1-k} \geq 0$, so applying $(17)$, we get

$$
f\left(\frac{x_{0}}{1-k}\right)=f\left(x_{0}+g\left(x_{0}\right) \frac{x_{0}}{1-k}\right)=f\left(x_{0}\right) f\left(\frac{x_{0}}{1-k}\right)=a f\left(\frac{x_{0}}{1-k}\right) \text {. }
$$


Since $Z_{f}=\emptyset$, this gives $a=1$. Furthermore, taking in (17) $x=x_{0}$, we obtain $f\left(x_{0}+k x\right)=f(x)$ for $x \in[0, \infty)$. Hence, by iteration, we get $f\left(k^{n} x+\right.$ $\left.x_{0} \sum_{i=0}^{n-1} k^{i}\right)=f(x)$ for $x \in[0, \infty), n \in \mathbb{N}$. Thus letting $n \rightarrow \infty$ and using the continuity of $f$, we obtain $f(x)=f\left(\frac{x_{0}}{1-k}\right)$ for $x \in[0, \infty)$. Consequently $f$ is constant and so, as $f(0)=1$, we get $f(x)=1$ for $x \in[0, \infty)$, which yields a contradiction. Therefore we have proved that $k>1$. Since $x_{0}$ is fixed arbitrarily this means that

$$
g(x) \geq 1 \text { for } x \in[0, \infty)
$$

Moreover, applying (17) with $x=x_{0}$, we get $f\left(x_{0}+k x\right)=a f(x)$ for $x \in[0, \infty)$. Thus, a straightforward calculation shows that a function $h:\left[\log _{k}\left(-\frac{x_{0}}{1-k}\right), \infty\right) \rightarrow \mathbb{R}$ given by

$$
h(x)=a^{-x} f\left(k^{x}+\frac{x_{0}}{1-k}\right),
$$

satisfies the condition $h(x+1)=h(x)$ for $x \in\left[\log _{k}\left(-\frac{x_{0}}{1-k}\right), \infty\right)$. Hence, as $h$ is continuous, there is a continuous 1-periodic function $\omega: \mathbb{R} \rightarrow \mathbb{R}$ such that $h(x)=\omega(x)$ for $x \in\left[\log _{k}\left(-\frac{x_{0}}{1-k}\right), \infty\right)$. Furthermore, in view of (19), we obtain

$$
f(x)=\left(x-\frac{x_{0}}{1-k}\right)^{\log _{k} a} \omega\left(\log _{k}\left(x-\frac{x_{0}}{1-k}\right)\right) \quad \text { for } x \in[0, \infty) .
$$

Since $\omega$ is a 1-periodic function, inserting into (17) $f$ of the form (20) and putting in the obtained in this way equality $k^{n} y$ instead of $y$ after a standard computation, we get

$$
\begin{aligned}
\left(g(x) y+\frac{x-\frac{x_{0}}{1-k}}{k^{n}}\right)^{\log _{k} a} \omega\left(\log _{k}\left(g(x) y+\frac{x-\frac{x_{0}}{1-k}}{k^{n}}\right)\right) \\
=\left(x-\frac{x_{0}}{1-k}\right)^{\log _{k} a} \omega\left(\log _{k}\left(x-\frac{x_{0}}{1-k}\right)\right) \\
\quad \cdot\left(y-\frac{x_{0}}{k^{n}}\right)^{\log _{k} a} \omega\left(\log _{k}\left(y-\frac{x_{0}}{1-k}\right)\right) \text { for } x, y \in[0, \infty), n \in \mathbb{N} .
\end{aligned}
$$

As $\omega$ is continuous, letting $n \rightarrow \infty$, in view of (18) and (20), we obtain

$$
f\left(g(x) y+\frac{x_{0}}{1-k}\right)=f(x) f\left(y+\frac{x_{0}}{1-k}\right) \quad \text { for } x \in[0, \infty), y \in\left(-\frac{x_{0}}{1-k}, \infty\right) .
$$

Therefore

$$
f(x)=\Psi(g(x)) \quad \text { for } x \in[0, \infty)
$$


where $\Psi: g([0, \infty)) \rightarrow \mathbb{R}$ is given by $\Psi(z)=\frac{f\left(z x_{0}+\frac{x_{0}}{1-k}\right)}{f\left(x_{0}+\frac{x_{0}}{1-k}\right)}$ for $z \in g([0, \infty))$. From (21) and (22) we obtain

$$
\bar{f}(z y)=\Psi(z) \bar{f}(y) \quad \text { for } z \in g([0, \infty)), y \in\left(-\frac{x_{0}}{1-k}, \infty\right),
$$

where $\bar{f}:\left(-\frac{x_{0}}{1-k}, \infty\right) \rightarrow \mathbb{R}$ is of the form $\bar{f}(z)=f\left(z+\frac{x_{0}}{1-k}\right)$. Since $k>1$, we have $\left(-\frac{x_{0}}{1-k}, \infty\right) \subset(0, \infty)$. Furthermore, as $f$ is nonconstant and continuous, so are $\bar{f}$ and $\Psi$. Note also that, in view of $(18)$ and $(22), g([0, \infty))$ is a nondegenerate interval contained in $[1, \infty)$. Thus int $g([0, \infty)) \times\left(-\frac{x_{0}}{1-k}, \infty\right)$ is a nonempty, open and connected subset of $(0, \infty)^{2}$. Therefore, applying [19, p. 311, Theorem 6] and [27, Corollary 2], from (23) we derive that there exist an $\alpha \in \mathbb{R} \backslash\{0\}$ and an $r \in \mathbb{R} \backslash\{0\}$ such that $\bar{f}(z)=\alpha z^{r}$ for $z \in\left(-\frac{x_{0}}{1-k}, \infty\right)$. Hence

$$
f(x)=\alpha\left(x-\frac{x_{0}}{1-k}\right)^{r} \quad \text { for } x \in(0, \infty) .
$$

As $f$ is continuous this implies that $1=f(0)=\alpha\left(-\frac{x_{0}}{1-k}\right)^{r}$. Thus $\alpha>0$ and

$$
f(x)=\left(1+\alpha^{\frac{1}{r}} x\right)^{r} \quad \text { for } x \in[0, \infty) .
$$

Inserting into (17) $f$ of the form (25) we get

$$
\left(1+\alpha^{\frac{1}{r}}(x+g(x) y)\right)^{r}=\left(1+\alpha^{\frac{1}{r}} x\right)^{r}\left(1+\alpha^{\frac{1}{r}} y\right)^{r} \quad \text { for } x, y \in[0, \infty) .
$$

Taking in the last equality $y=1$ and using the injectivity of the map $(0, \infty) \ni$ $x \mapsto x^{r} \in(0, \infty)$ after a straightforward calculation we obtain $g(x)=1+\alpha^{\frac{1}{r}} x$ for $x \in[0, \infty)$, which together with (25) gives (iv) with $c:=\alpha^{\frac{1}{r}}$.

Case (II) According to Remark 2.2, for every $x \in[0, \infty)$, we have either $g^{(x)}(z)<0$ or $g^{(x)}(z) \in Z_{f}=\{z\}$. Hence, for every $x \in[0, \infty)$, we get either $g(x)<-\frac{x}{z}$ or $g(x)=1-\frac{x}{z}$. Since $g$ is continuous this means that either

$$
g(x)<-\frac{x}{z}<0 \quad \text { for } x \in[0, \infty)
$$

or

$$
g(x)=1-\frac{x}{z} \quad \text { for } x \in[0, \infty)
$$

If (26) holds then $-\frac{z}{g(z)}>0$ and $z+g(z)\left(-\frac{z}{g(z)}\right)=0$, so applying (4) with $x=z$ and $y=-\frac{z}{g(z)}$, we obtain $1=f(0)=f\left(z+g(z)\left(-\frac{z}{g(z)}\right)\right)=f(z) f(-$ $\left.\frac{z}{g(z)}\right)=0$, which gives a contradiction. Therefore (27) is valid. Next, note that if $x \in[0, \infty)$ and $y \in[0, z]$, in view of $(27)$, we get $x+g(x) y=x\left(1-\frac{y}{z}\right)+y \geq 0$. Thus (4) and (27) imply

$$
f\left(x+y-\frac{x y}{z}\right)=f(x) f(y) \text { for } x \in[0, \infty), y \in[0, z] .
$$


Let $f_{1}:[0,1] \rightarrow \mathbb{R}$ be given by

$$
f_{1}(x)=f(z(1-x)) \text { for } x \in[0,1] .
$$

Then $f_{1}$ is continuous and nonconstant [because $f_{1}(0)=f(z)=0$ and $f_{1}(1)=$ $f(0)=1]$. Moreover, in view of $(28)$, we get

$$
\begin{aligned}
f_{1}(u v) & =f(z(1-u v))=f\left(z(1-u)+z(1-v)-\frac{1}{z} z(1-u) z(1-v)\right) \\
& =f(z(1-u)) f(z(1-v))=f_{1}(u) f_{1}(v) \quad \text { for } u, v \in[0,1] .
\end{aligned}
$$

Therefore, applying [19, p.311, Theorem 6], we conclude that there exists an $r \in(0, \infty)$ such that

$$
f_{1}(u)=u^{r} \quad \text { for } \quad u \in[0,1]
$$

Hence, in view of (29),

$$
f(x)=\left(1-\frac{x}{z}\right)^{r} \quad \text { for } \quad x \in[0, z] .
$$

Furthermore, making use of $(28)$, for every $x \in(z, \infty)$ and $y \in(0, z)$, we obtain

$$
\begin{aligned}
f\left(z\left(1+\left(\frac{x}{z}-1\right)\left(1-\frac{y}{z}\right)\right)\right) & =f\left(x+y-\frac{x y}{z}\right)=f(x) f(y) \\
& =f\left(z\left(1+\left(\frac{x}{z}-1\right)\right)\right) f\left(z\left(1-\left(1-\frac{y}{z}\right)\right)\right) .
\end{aligned}
$$

Thus

$$
f_{2}(u v)=f_{2}(u) f_{1}(v) \quad \text { for } \quad u \in(0, \infty), v \in(0,1),
$$

where $f_{2}:(0, \infty) \rightarrow \mathbb{R}$ is given by

$$
f_{2}(x)=f(z(1+x)) \text { for } x \in(0, \infty) .
$$

Since $f_{2}$ is continuous, $(0, \infty) \times(0,1)$ is an open and connected subset of $(0, \infty)^{2}$ and $f_{2}(x) \neq 0$ for $x \in(0, \infty)$ (because $\left.Z_{f}=\{z\}\right)$, applying [19, p.311, Theorem 6] and [27, Corollary 2], from (30) and (32) we derive that

$$
f_{2}(u)=\alpha u^{r} \text { for } u \in(0, \infty)
$$

with some $\alpha \in \mathbb{R} \backslash\{0\}$. Furthermore, in view of $(27)$, we get $2 z+g(2 z) \cdot 2 z=0$. Thus, as $f(0)=1$, putting in (4) $x=y=2 z$, we obtain $f(2 z)^{2}=1$. Hence (33) and (34) imply that $\alpha=f_{2}(1)=f(2 z) \in\{-1,1\}$. If $\alpha=1$ then from (33) and (34) we deduce that $f(x)=\left(\frac{x}{z}-1\right)^{r}$ for $x \in(z, \infty)$, which together with (31) gives $f(x)=\left|1-\frac{x}{z}\right|^{r}$ for $x \in[0, \infty)$. If $\alpha=-1$ then from (33) and (34) follows that $f(x)=-\left(\frac{x}{z}-1\right)^{r}$ for $x \in(z, \infty)$. Thus, in view of $(31)$, we get $f(x)=\left|1-\frac{x}{z}\right|^{r} \operatorname{sgn}\left(1-\frac{x}{z}\right)$ for $x \in[0, \infty)$. Hence, as $-\frac{1}{z}<0$, taking into account (27), we obtain (v) with $c:=-\frac{1}{z}$.

Case (III) According to Remark 2.2, for every $x \in[0, z)$, either $g^{(x)}(z)<0$ or $g^{(x)}(z) \in Z_{f}=[z, \infty)$. Thus, for every $x \in[0, z)$, we have either $g(x)<-\frac{x}{z}$ 
or $g(x) \geq 1-\frac{x}{z}$. Therefore, using the continuity of $g$ we conclude that either $g(x)<-\frac{x}{z}$ for $x \in[0, z)$ or

$$
g(x) \geq 1-\frac{x}{z}>0 \quad \text { for } x \in[0, z) .
$$

Arguing as in the previous case we exclude the first possibility. So, (35) is valid. Suppose that $g(x)>1-\frac{x}{z}$ for some $x \in[0, z)$. Then $g^{(x)}(0)=x<$ $z<x+g(x) z=g^{(x)}(z)$. As $g^{(x)}$ is continuous this means that there is a $y \in(0, z)$ such that $g^{(x)}(y)=z \in Z_{f}$. Since $x, y \in(0, z)$ and $(0, z) \cap Z_{f}=\emptyset$, this contradicts (10). Consequently, using again the continuity of $g$, we get

$$
g(x)=1-\frac{x}{z} \quad \text { for } x \in[0, z] .
$$

Furthermore, similarly as in the previous case, we obtain that the function $f_{1}:[0,1] \rightarrow \mathbb{R}$ given by (29) is nonconstant, continuous and it satisfies the equation $f_{1}(u v)=f_{1}(u) f_{1}(v)$ for $u, v \in[0,1]$. Hence, applying [19, p.311, Theorem 6], we obtain (31) with some $r \in(0, \infty)$. Suppose that $g\left(x_{0}\right)<0$ for some $x_{0} \in[z, \infty)$. Then $\frac{\frac{1}{2} z-x_{0}}{g\left(x_{0}\right)}>0$ and $g^{\left(x_{0}\right)}\left(\frac{\frac{1}{2} z-x_{0}}{g\left(x_{0}\right)}\right)=\frac{1}{2} z>0$. Thus, in view of (9), we get $f\left(\frac{1}{2} z\right)=f\left(x_{0}\right) f\left(\frac{\frac{1}{2} z-x_{0}}{g\left(x_{0}\right)}\right)$. On the other hand, as $Z_{f}=[z, \infty)$, we have $f\left(\frac{1}{2} z\right) \neq 0$ and $f\left(x_{0}\right)=0$. This yields a contradiction. Therefore $g(x) \geq 0$ for $x \in[z, \infty)$ and so, taking into account (31) and (36), we obtain (vi) with $c:=-\frac{1}{z}$ and $\phi:=g_{\mid[z, \infty)}$.

The converse is easy to check.

From Theorem 3.1 we derive the following result (cf. [24]).

Corollary 3.2. A continuous function $f:[0, \infty) \rightarrow \mathbb{R}$ satisfies $E q$. (3) if and only if one of the following possibilities holds:

(i) $f(x)=0$ for $x \in[0, \infty)$;

(ii) there exist a $c \in \mathbb{R}$ such that

$$
f(x)=1+c x \text { for } x \in[0, \infty) ;
$$

(iii) there exist a $c \in(-\infty, 0)$ such that

$$
f(x)=\max \{1+c x, 0\} \text { for } x \in[0, \infty) .
$$

It is well known (cf. e.g. $[1,13]$ ) that a continuous function $F: \mathbb{R} \rightarrow \mathbb{R}$ satisfies equation

$$
F(x+F(x) y)=F(x) F(y) \text { for } x, y \in \mathbb{R}
$$

if and only if $F(x)=0$ for $x \in \mathbb{R}$ or $F(x)=1+c x$ for $x \in \mathbb{R}$ with some $c \in \mathbb{R}$ or $F(x)=\max \{1+c x, 0\}$ with some $c \in \mathbb{R} \backslash\{0\}$. Therefore from Corollary 3.2 we derive the following extension result for (3).

Corollary 3.3. If a continuous function $f:[0, \infty) \rightarrow \mathbb{R}$ satisfies Eq. (3) then there exists a continuous function $F: \mathbb{R} \rightarrow \mathbb{R}$ satisfying (39) such that

$$
f(x)=F(x) \text { for } x \in[0, \infty) .
$$


The next example shows that in the case of (4) the counterpart of Corollary 3.3 does not hold.

Example. Let $g, f:[0, \infty) \rightarrow \mathbb{R}$ be given by $g(x)=|1-x|$ for $x \in[0, \infty)$ and $f(x)=\max \{1-x, 0\}$ for $x \in[0, \infty)$. Then $f$ and $g$ are continuous and the pair $(f, g)$ satisfies Eq. (4). However, in view of Proposition 2.1, there is no pair $(F, G)$ of continuous functions $F, G: \mathbb{R} \rightarrow \mathbb{R}$ satisfying (6) such that (40) holds and $g(x)=G(x)$ for $x \in[0, \infty)$.

Now, applying Theorem 3.1, we determine continuous solutions of Eq. (5).

Theorem 3.4. Assume that $F, G: \mathbb{R} \rightarrow \mathbb{R}$ are continuous functions. Then the pair $(F, G)$ satisfies Eq. (5) if and only if one of the subsequent possibilities holds:

(a) $F(x)=0$ for $x \in \mathbb{R}$;

(b) $F(x)=1$ for $x \in \mathbb{R}$;

(c) $F(x)=0$ for $x \in[0, \infty)$ and $G([0, \infty)) \subset[0, \infty)$;

(d) $F(x)=1$ for $x \in[0, \infty)$ and $G([0, \infty)) \subset[0, \infty)$;

(e) $G(x)=1$ for $x \in[0, \infty)$ and there exists a $c \in \mathbb{R} \backslash\{0\}$ such that $F(x)=$ $e^{c x}$ for $x \in[0, \infty)$

(f) there exist a $c \in(0, \infty)$ and an $r \in \mathbb{R} \backslash\{0\}$ such that

$$
G(x)=1+c x \quad \text { for } \quad x \in[0, \infty)
$$

and

$$
F(x)=(1+c x)^{r} \quad \text { for } \quad x \in[0, \infty) ;
$$

(g) there exist a $c \in(-\infty, 0)$ and an $r \in(0, \infty)$ such that $G$ is of the form (41) and $F$ is either of the form (7) or (8);

(h) there exist a $c \in(-\infty, 0)$, an $r \in(0, \infty)$ and a continuous function $\phi$ : $\left[-\frac{1}{c}, \infty\right) \rightarrow[0, \infty)$ with $\phi\left(-\frac{1}{c}\right)=0$ such that

$$
G(x)= \begin{cases}1+c x & \text { for } x \in\left[0,-\frac{1}{c}\right) \\ \phi(x) & \text { for } x \in\left[-\frac{1}{c}, \infty\right)\end{cases}
$$

and

$$
F(x)=(\max \{1+c x, 0\})^{r} \quad \text { for } \quad x \in[0, \infty) .
$$

Proof. It is easy to check that if one of the possibilities $(a)-(h)$ holds then the pair $(F, G)$ satisfies (5). So, assume that the pair $(F, G)$ satisfies $(5)$. Let $f:=F_{\mid[0, \infty)}$ and $g:=G_{\mid[0, \infty)}$. Then $f$ and $g$ are continuous and the pair $(f, g)$ satisfies (4). Therefore, according to Theorem 3.1, one of the cases $(i)$-(vi) holds. Obviously (iii) implies $(e),(i v)$ leads to $(f)$ and $(v i)$ yields $(h)$. If $(i)$ holds then $F(x)=0$ for $x \in[0, \infty)$, so if $G\left(x_{0}\right)<0$ for some $x_{0} \in[0, \infty)$, then in view of $(5)$, we get $F\left(x_{0}+G\left(x_{0}\right) y\right)=0$ for $y \in[0, \infty)$. Since $\left\{x_{0}+G\left(x_{0}\right) y \mid y \in\right.$ $[0, \infty)\}=\left(-\infty, x_{0}\right]$ this means that $F(x)=0$ for $\left(-\infty, x_{0}\right]$. Consequently, either $(a)$ or $(c)$ holds. Similarly, if $(i i)$ is valid then $F(x+G(x) y)=1$ for 
$x, y \in[0, \infty)$. So, if $G\left(x_{0}\right)<0$ for some $x_{0} \in[0, \infty)$, then arguing as previously we conclude that $F(x)=1$ for $x \in\left(-\infty, x_{0}\right]$. Thus either $(b)$ or $(d)$ holds.

It remains to consider the case where $(v)$ is valid. First note that $(14)$ implies (41). Next, as $c \in(-\infty, 0)$, we have

$$
F\left(-\frac{2}{c}\right)=f\left(-\frac{2}{c}\right)= \begin{cases}1 & \text { whenever }(15) \text { holds } \\ -1 & \text { whenever }(16) \text { holds }\end{cases}
$$

Furthermore $-\frac{2}{c}-x>0$ for $x \in(-\infty, 0)$ and, in view of $(41), G\left(-\frac{2}{c}\right)=-1$. Therefore, applying (5), we obtain

$$
\begin{aligned}
F(x) & =F\left(-\frac{2}{c}+(-1)\left(-\frac{2}{c}-x\right)\right)=F\left(-\frac{2}{c}+G\left(-\frac{2}{c}\right)\left(-\frac{2}{c}-x\right)\right) \\
& =F\left(-\frac{2}{c}\right) F\left(-\frac{2}{c}-x\right)=f\left(-\frac{2}{c}\right) f\left(-\frac{2}{c}-x\right) \text { for } x \in(-\infty, 0) .
\end{aligned}
$$

Hence, making use of (42), we get $F(x)=f\left(-\frac{2}{c}-x\right)=|1+c x|^{r}$ for $x \in$ $(-\infty, 0)$, whenever (15) holds; and $F(x)=-f\left(-\frac{2}{c}-x\right)=|1+c x|^{r} \operatorname{sgn}(1+c x)$ for $x \in(-\infty, 0)$, whenever (16) holds. Thus $(g)$ is valid.

From Theorem 3.4 one can deduce the following result (cf. [3]).

Corollary 3.5. A continuous function $f: \mathbb{R} \rightarrow \mathbb{R}$ satisfies Eq. (2) if and only if one of the following possibilities holds:

(i) $f(x)=0$ for $x \in[0, \infty)$;

(ii) there exists a $c \in[0, \infty)$ such that (37) holds;

(iii) there exists a $c \in(-\infty, 0)$ such that either (38) holds or $f(x)=1+$ cx for $x \in \mathbb{R}$.

As a consequence of Corollary 3.5 we have the extension result for Eq. (2).

Corollary 3.6. If a continuous function $f: \mathbb{R} \rightarrow \mathbb{R}$ satisfies Eq. (2) then there exists a continuous function $F: \mathbb{R} \rightarrow \mathbb{R}$ satisfying (39) such that (40) holds.

Remark 3.7. A counterpart of Corollary 3.6 does not hold for (5). To see this, it is enough to take $G: \mathbb{R} \rightarrow \mathbb{R}$ of the form $G(x)=|1-x|$ for $x \in \mathbb{R}$ and $F: \mathbb{R} \rightarrow \mathbb{R}$ given by $F(x)=\max \{1-x, 0\}$ for $x \in \mathbb{R}$.

\section{Acknowledgements}

This work was partially supported by the Centre for Innovation and Transfer of Natural Sciences and Engineering Knowledge. The second author thank Professor Sergei Trofimchuk for stimulating discussions and useful suggestions.

Open Access. This article is distributed under the terms of the Creative Commons Attribution License which permits any use, distribution, and reproduction in any medium, provided the original author(s) and the source are credited. 


\section{References}

[1] Aczél, J., Dhombres, J.: Functional equations in several variables. In: Encyclopedia of Mathematics and its Applications, vol. 31. Cambridge University Press, Cambridge (1989)

[2] Aczél, J., Gołąb, S.: Remarks on one-parameter subsemigroups of the affine group and their homo- and isomorphisms. Aequationes Math. 4, 1-10 (1970)

[3] Aczél, J., Schwaiger, J.: Continuous solutions of the Gołąb-Schinzel equation on the nonnegative reals and on related domains. Österr. Akad. Wiss. Math.-Natur. Kl. Sitzungsber. II 208, 171-177 (1999)

[4] Aichinger, E., Farag, M.: On when the multiplicative center of a near-ring is a subnear-ring. Aequationes Math. 48, 46-59 (2004)

[5] Brillouët-Belluot, N., Brzdȩk, J.: On continuous solutions and stability of a conditional Gołąb-Schinzel equation. Publ. Math. Debrecen 72, 441-450 (2008)

[6] Brillouët-Belluot, N., Brzdȩk, J., Chudziak, J.: On continuous solutions of a class of conditional equations. Publ. Math. Debrecen 75, 11-22 (2009)

[7] Brillouët, N., Dhombres, J.: Équations fonctionnelles et recherche de sousgroupes. Aequationes Math. 31, 253-293 (1986)

[8] Brzdęk, J.: On continuous solutions of a conditional Gołąb-Schinzel equation. Anz. Österreich. Akad. Wiss. Math.-Natur. Kl. 138, 3-6 (2001)

[9] Brzdęk, J.: The Gołąb-Schinzel equation and its generalizations. Aequationes Math. 70, 14-24 (2005)

[10] Brzdȩk, J., Mureńko, A.: On a conditional Gołąb-Schinzel equation. Arch. Math. (Basel) 85, 503-511 (2005)

[11] Chudziak, J.: Semigroup-valued solutions of the Gołąb-Schinzel type functional equation. Abh. Math. Sem. Univ. Hamburg 76, 91-98 (2006)

[12] Chudziak, J.: Associative solutions of a composite generalization of addition formulae. Acta Math. Hungar. 117, 141-152 (2007)

[13] Gołąb, S., Schinzel, A.: Sur l'équation fonctionnelle $f(x+y f(x))=f(x) f(y)$. Publ. Math. Debrecen 6, 113-125 (1959)

[14] Ilse, D., Lehmann, I., Schulz, W.: Gruppoide und Funktionalgleichungen. VEB Deutscher Verlag der Wissenschaften, Berlin (1984)

[15] Jabłońska, E.: Continuous on rays solutions of an equation of the Gołąb-Schinzel type. J. Math. Anal. Appl. 375, 223-229 (2011)

[16] Jabłońska, E.: On continuous solutions of an equation of the Gołąb-Schinzel type. Bull. Aust. Math. Soc. 87, 10-17 (2013)

[17] Jabłońska, E.: On locally bounded above solutions of an equation of the GołąbSchinzel type. Aequationes Math. doi:10.1007/s00010-013-0195-9

[18] Kahlig, P., Matkowski, J.: A modified Gołąb-Schinzel equation on a restricted domain (with applications to meteorology and fluid mechanics). Österr. Akad. Wiss. Math.-Natur. Kl. Sitzungsber. II. 211, 117-136 (2002)

[19] Kuczma, M.: An Introduction to the Theory of Functional Equations and Inequalities, Państwowe Wydawnictwo Naukowe, Uniwersytet Śląski, WarszawaKraków-Katowice (1985) 
[20] Luneburg, H., Plaumann, P.: Die Funktionalgleichung von Gołąb und Schinzel in Galoisfeldern. Arch. Math. (Basel) 28, 55-59 (1977)

[21] Mureńko, A.: On solutions of the Gołąb-Schinzel equation. Int. J. Math. Math. Sci. 27, 541-546 (2001)

[22] Mureńko, A.: On solutions of a conditional generalization of the Gołąb-Schinzel equation. Publ. Math. Debrecen 63, 693-702 (2003)

[23] Plaumann, P., Strambach, S.: Zweidimensionale Quasialgebren mit Nullteilern. Aequationes Math. 15, 249-264 (1977)

[24] Reich, L.: Über die stetigen Lösungen der Gołąb-Schinzel-Gleichung auf $\mathbb{R}$ und auf $\mathbb{R}_{\geq 0}$, Österr. Akad. Wiss. Math.-Natur. Kl. Sitzungsber. II. 208, 165-170 (1999)

[25] Reich, L.: Über die stetigen Lösungen der Gołąb-Schinzel-Gleichung auf $\mathbb{R}_{\geq 0}$, Anz. Österreich. Akad. Wiss. Math.-Natur. Kl. 138, 7-12 (2001)

[26] Sablik, M.: A conditional Gołąb-Schinzel equation. Anz. Österreich. Akad. Wiss. Math.-Natur. Kl. 137, 11-15 (2000)

[27] Sobek, B.: Pexider equation on a restricted domain. Demonstratio Math. 43, 8188 (2010)

Jacek Chudziak

Faculty of Mathematics and Natural Sciences

University of Rzeszów

Prof. St. Pigonia 1

35-310 Rzeszow

Poland

e-mail: chudziak@ur.edu.pl

Zdeněk Kočan

Mathematical Institute in Opava

Silesian University in Opava

Na Rybničku 1

74601 Opava

Czech Republic

e-mail: zdenek.kocan@math.slu.cz

Received: December 30, 2013.

Accepted: February 4, 2014. 